\title{
EEG features of sleepy state analysis
}

\author{
Mu Zhen-dong \\ College of Information Engineering, Jiangxi College of Technology, Jiangxi Nanchang 330098
}

\section{Keywords: Fatigue detection; EEG; Quantify}

\begin{abstract}
Doze reflect a physiological brain state of extreme fatigue, fatigue effect will be affected by the state of the work; some work would even human life crisis. In this paper, the use of EEG as a tool, designed by the methods described herein to quantify fatigue and brain fatigue realized through homogenization methods for quantitative indicators of fatigue under different states of implementation monitoring.
\end{abstract}

\section{Introduction}

Doze fatigue is a physiological response of the brain, but also a progressive state, and now at home and abroad have conducted standardized classification of sleep, but a state of drowsiness yet a unified standard, people from the excitement - the norm - Burnout - sleepy - No significant between very sleepy classification boundaries, and therefore do not have a good way to monitor the sleepy, sleepy for some long monotonous work state, because it makes a lot of privacy and progressive forms of accidents in the know do not sleep occurs, such as driving, fatigue driving caused the accident because the driver was not under a state of drowsiness timely reminder happen, so sleepy state study has important application value.

EEG findings indicate that many of the external manifestations can get results from the cerebral cortex function tests, EEG responses to external, can be amplitude, frequency, etc. to represent a variety of features, the findings show that when people in different mental state, when the basic characteristics of brain waves with the corresponding stable discontinuous variability, the variability can be described by a certain function.

With the advent of the development of electronic technology, intelligent terminals and mobile EEG acquisition devices, the driver gave to provide technical support for research sleepy EEG-based monitoring methods, signal analysis method on the driver's brain real-time detection signals read by intelligent terminal EEG and calculated by setting a threshold value to remind the driver over and driving interventions in order to reduce the accident rate of fatigue driving.

Although research sleepy and drowsy state are rare, but closely related research and sleepy, but a lot of attention, for the attention of the most successful research is carried out through the EEG time-frequency domain analysis, extraction energy is one of the EEG spectrum analysis of different bands, from the composition ratio can determine the degree of attention.

Sleepy similar attention, but the attention is more complex than research, therefore, the present invention is not only the traditional method using frequency domain analysis when analyzed from different energy band spectral components, but also from the EEG and EEG phase itself On analyzed separately second order blind identification, phase synchronization and energy entropy method for EEG analysis, from the analysis of different aspects of a state of drowsiness, which can more accurately reflect the relationship between brain activity and sleepy.

In this paper, EEG as a research tool, designed to capture the different state of EEG frequency components to calculate the fatigue state using the method designed in this paper, by analyzing the relationship between these states and got brainwave frequency components, the establishment of the state of quantifying fatigue relationship, then this quantitative results homogenized human tendency to fatigue, providing real-time EEG may fatigue detection technology. 


\section{Method}

Subjects in the driving environment sub-state segment EEG acquisition, the state is divided into segments awake, has been significantly dozing state, and shallow sleep, and then several times in normal subjects from awake to sleep with, then EEG acquisition to awake from sleep, the whole collection of different sex experiment, the subjects of different ages EEG data to establish a database related EEG, EEG technology acquisition parameters include the acquisition process The sampling frequency is $128 \mathrm{~Hz}$, were $1 \sim 49 \mathrm{~Hz}$ band-pass filtering EEG data every three seconds as a storage unit.

The driver EEG database, for each valid sample intercepts a sample of 384 points as the segment consecutive samples taken as data analysis section of an object, we mainly use gravity band feature extraction method described herein . EEG signal band composed of four main components: $\delta, \vartheta, \alpha, \beta$, when sober, when human consciousness, $\alpha, \beta$ predominant component, when people are not awake consciousness, $\delta, \vartheta$ accounted for the main ingredient. EEG bands corresponding to different frequency ranges are: $\alpha$ is $8 \sim 12 \mathrm{~Hz}, \beta$ is $13 \sim 30 \mathrm{~Hz}, \delta$ is $4 \sim 7 \mathrm{~Hz}, \vartheta$ is $1 \sim 3 \mathrm{~Hz}$.

Use the following formula to calculate the proportion of $\mathrm{S}$ different frequencies in different states:

$$
S(\omega)=\frac{\int_{\omega 1}^{\omega 2} A(t) d t}{\int_{1}^{50} A(t) d t}
$$

Wherein $\delta, \vartheta, \alpha, \beta$ represents four bands, $\omega 1, \omega 2$ represents lower and upper bands, $A(t)$ represents a function of the AR model EEG transformed.

Using the following formula were the subjects EEG database of valid data to calculate the proportion of different bands $\bar{S}(\omega)$.

$$
\bar{S}(\omega)=\frac{\sum_{1}^{5} S(\omega)}{n}
$$

Where $\mathrm{n}$ represents the number of valid samples.

Subjects using the following formula to calculate the degree of drowsiness AL:

$$
A L=\frac{S(\alpha)+S(\beta)}{S(\delta)+S(\vartheta)}
$$

By the above method to calculate the proportion of frequency bands can be in different states under different subjects, according to the results, select the frequency characteristics of the subjects brainwave, this can form the subject of fatigue signatures - sleepy extent corresponding AL relationship, this feature database can be represented as a plurality of vectors we (sample), with each vector represented as $\mathrm{EA}=(\mathrm{a} 1, \mathrm{a} 2, \mathrm{a} 3,,,$, , an $)$.

Setting subjects awake EEG signature sequence is expressed as EA = (a1, a2, a3 ,,,, an), then the number of successful participants in the EEG database, better sample characteristics formed into a sober state matrix EA = (ea1, ea2, ea3 ,, ean) Similarly EEG features available subjects and the subjects in the matrix EK asleep at significantly drowsy state EEG state signal characteristic matrix ES, above EEG features are characteristic of brain electrical driving state 3 seconds for the next period of time, assuming that collected under normal EEG is marked as CME. CME conducted on normal EEG interception once every three seconds per slide back 128 data points (one second), and then follow the above method for feature extraction to obtain EEG data segment features the three seconds of CMEt, were calculated CMEt and EA, EK, ES matching degree, the degree of matching the value of $\mathrm{x}$ is calculated as:

$$
\mathrm{x}=\left|\lambda E A-C E M t^{T}\right|
$$


Here is the characteristic adjustment coefficient, when the results of an $\mathrm{x}$ in the set threshold range of time, you can determine which segments of EEG match to awake subjects, the same method to calculate the normal state corresponding to driving the process state and the EK ES state, continuous EEG such original normal is 3 seconds, the length of the time window to be segmented into discrete EEG, in each of the EEG time series and is continuous, and it varies from waking to sleeping continuous state of human brain responses corresponding to the same time as EA, EK and ES corresponding to the continuous piecewise normal EEG features a mapping relationship with EA as a starting point, ES for the end, you can determine the next course of driving drowsy state driver characteristics. By contrast, after the establishment of a continuous electrical brain signatures subjects under continuous driving state map, the corresponding EEG quantified according to different subjects, collected EEG is different, so between EA and ES The map is not the same width, and some subjects between EA and ES, which corresponds to the width of the normal 100, and some subjects that have a width 80 , in order to facilitate the participants were reminded that this method unification 50 for the measurement methods, when the width of less than 50 subjects, the use of many-uniform mapping, when the width is greater than 50, then the uniform of many mapping.

4, the establishment of the subject by the above method of mapping feature library sleepy state, because there is no specific reaction of the subjects themselves, so the EEG feature extraction method commonly contain a lot of redundancy and noise, for the individual subject test is concerned, with fewer features may also play a very good effect classification, and fewer features means that the faster speed, so this method for each subject, to establish their own monitor.

\section{Results}

In this paper, the method described above for 15 subjects fatigue detection, were set up 5 fatigue detection points, so that fatigue of these 15 subjects were divided into $(0-1,1-2,2-3,3-4,4-5)$ five stages and then these five stages of fatigue uniform, each subject to select 300 samples available for these samples to accurately obtain the status of a subject sample shown below :
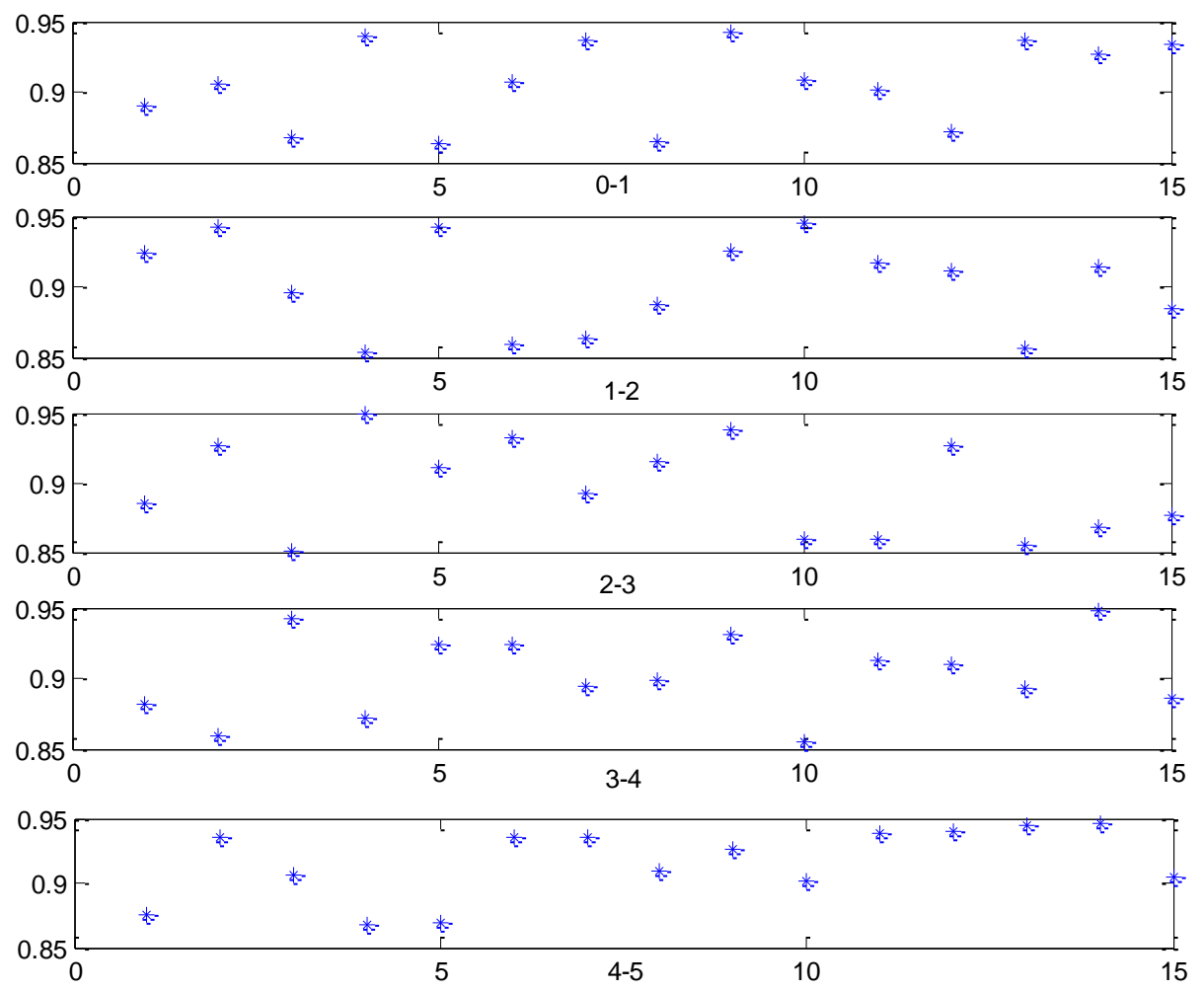
Figure 1 show that, for a fatigue stage, fit 15 subjects in more than 80 percent, the data show that the use of brain Telecom fatigue detection is feasible; this method can be a good measure to achieve continuous fatigue shown.

\section{Acknowledgements}

This work was financially supported by project of Technology Department of Jiangxi Province [No 20143BBM26048] and project of Jiangxi University of Technology [No. xtcx201312].

\section{References}

[1] Jap B T, Lal S, Fischer P, et al. Using EEG spectral components to assess algorithms for detecting fatigue[J]. Expert Systems with Applications, 2009, 36(2): 2352-2359.

[2] Wang Q, Yang J, Ren M, et al. Driver fatigue detection: a survey[C]//Intelligent Control and Automation, 2006. WCICA 2006. The Sixth World Congress on. IEEE, 2006, 2: 8587-8591.

[3] Kar S, Bhagat M, Routray A. EEG signal analysis for the assessment and quantification of driver's fatigue[J]. Transportation research part F: traffic psychology and behaviour, 2010, 13(5): 297-306.

[4] Zhao J, Pan P, Fu W, et al. Research on mental fatigue detection based on portable EEG acquisition equipment[C]//Intelligent Control and Automation (WCICA), 2014 11th World Congress on. IEEE, 2014: 5143-5146. 